\title{
Efecto de la modalidad de juego en baloncesto (5vs.5 y 3vs.3) sobre conductas motrices y psicológicas en alumnado de 9-11 años \\ Effect of game form (5vs.5 and 3vs.3) on motor and psychological behaviours in under-11 basketball \\ Francisco López-Herrero, Jose Luis Arias-Estero \\ Universidad Católica San Antonio de Murcia (España)
}

\begin{abstract}
Resumen. El objetivo fue comprobar si la modalidad de juego 3vs.3, en comparación con la $5 v s .5$ en baloncesto extraescolar jugado por niños de entre 9 y 11 años, favoreció un aumento de: (a) número de posesiones de balón, (b) número de pases, (c) eficacia en las posesiones, (d) disfrute, (e) competencia percibida, (f) intención de práctica futura, (g) emociones positivas, y (h) preferencia de los participantes. Comenzaron participando 42 niños $(\mathrm{M}=9.89$, DT $=.832)$, pero debido a los criterios de inclusión exigidos, los participantes finales fueron 18. Se utilizó la metodología observacional mediante un diseño ideográfico, seguimiento, multidimensional e intrasesional, con un muestreo temporal por puntos de tiempo para evaluar las dos modalidades de juego (5vs.5 y 3vs.3). Se empleó un diseño cuasi-experimental, a través del cual se comprobó el efecto de las dos modalidades de juego sobre las variables no observables. Los datos se obtuvieron a través de la filmación de los mini-partidos y su respectiva observación (número de posesiones de balón, número de pases y eficacia de las posesiones), empleando cuestionarios (disfrute, competencia percibida, intención de práctica futura y estado emocional), y a través de dos preguntas ad hoc por escrito (preferencia de los participantes). Los resultados mostraron valores más elevados a favor de la modalidad 3vs.3 en: (a) número de posesiones de balón, (b) número de pases, (c) eficacia de las posesiones, (d) emociones positivas, (e) emociones neutras y (f) preferencia de los participantes. Para favorecer la implicación en el juego, una práctica de calidad, las emociones positivas, y a la vez satisfacer las preferencias de los niños, se debería optar por la modalidad de juego 3vs.3. Palabras clave: Minibásquet, minibasket, baloncesto de iniciación, modificación de regla, juegos reducidos, inteligencia emocional, análisis de juego, intencionalidad de práctica futura.
\end{abstract}

\begin{abstract}
The purpose of the study was to know whether the 3vs.3 game form in comparison to the $5 \mathrm{vs} .5$ game form in under- 11 extra scholar basketball let an increase in: (a) number of ball possessions, (b) number of passes, (c) efficacy of the possessions, (d) enjoyment, (e) perceived competence, (f) intention to be physically active, (g) positive emotions, and (h) preference of the participants. Forty-two boys were involved in the study, but only 18 ended their participation due to the inclusion criteria. The observational methodology was used throughout an ideographic, follow-up, multidimensional, and intra-session design type. The sample technique to assess the two game forms (5vs.5 and 3vs.3) was temporary by time points. A quasi-experimental design was also used to explore the effect of the two game forms on the non-observable variables. Data were collected observing the videos of the matches (number of ball possessions, number of passes, efficacy of the possessions), using questionnaires (enjoyment, perceived competence, intention to be physically active, positive emotions), and two ad hoc written questions (preference of the participants). The results showed higher values in the $3 \mathrm{vs} .3$ game form in comparison to the $5 \mathrm{vs} .5$ game form in: (a) number of ball possessions, (b) number of passes, (c) efficacy of the possessions, (e) positive emotions, and (f) preference of the participants. The 3vs.3 game form should be used to favour players' game implication, successful participation, positive emotions, as well as, to satisfy their preferences.
\end{abstract}

Key words: Mini-basketball, youth basketball, rule modification, game analysis, modified games, small-sided games, emotional intelligence, intention to be physically active.

\section{Introducción}

El baloncesto jugado por niños de entre 9 y 11 años, también denominado minibásquet, se creó como una adaptación del baloncesto a las características de los niños de esas edades (Tous, 1999). Desde su creación han sido varios los estudios que han realizado propuestas teóricas y empíricas para mejorar dicha adecuación (e.g., American Sport Education Program, 2006; Arias-Estero, Argudo, \& Alonso, 2009, 2012, 2018; Carrillo \& Rodríguez, 2004; Giménez \& SáenzLópez, 2004; Mayol, Leon, \& Cardenas, 2016; Mitjana, 2000; Piñar, 2005). Algunas de estas propuestas han sido tenidas en cuenta por los organismos que regulan el deporte, aunque muchas otras siguen sin considerarse. Dentro de los cambios no implementados, parece transcendental reducir el número de jugadores participantes (Arias-Estero, Argudo, \&Alonso, 2011a; Martínez-Fernández, García, \& Ibáñez, 2015; Piñar, Cárdenas, Alarcón, Escobar, \& Torre, 2009; Tallir,

Fecha recepción: 18-08-18. Fecha de aceptación: 17-04-19 Jose Luis Arias-Estero

jlarias@ucam.edı
Phillipaerts, Valcke, Musch, \& Leonor, 2012). Dado que el número de jugadores debe estar determinado por la finalidad con la que se practica un deporte en iniciación deportiva (Carron, Widmeyer, \& Brawley, 1989), se considera plenamente justificado el estudio de la modalidad de juego 3vs.3 en aras a aportar conocimiento científico, que ayude a tomar decisiones sobre el número de jugadores participantes en baloncesto de 9 a 11 años. De este modo se contribuiría a crear contextos de práctica que mejorasen las experiencias motrices de los niños en cuanto a cantidad y éxito en la participación e interés por seguir practicando el deporte (Arias-Estero, Argudo, \& Alonso, 2011b).

Son varios los estudios realizados en etapas de formación en otros deportes de equipo en los que se analizó el efecto de la reducción del número de jugadores sobre diferentes variables. En fútbol se encontró que la modalidad 3vs.3 producía más pases, entradas, regates y situaciones de gol respecto a la modalidad 6vs.6 (Katis \& Kellis, 2009). Similares resultados hallaron Da Silva et al. (2011) tras comparar diferentes modalidades de juego (3vs.3, 4vs.4 y 5vs.5). Esta modalidad también fue estudiada en entrenamiento para mejorar aspectos técnico-tácticos y físicos en jugadores jó- 
venes de fútbol, obteniendo mejoras en la conducción, regate, ataque colectivo, agilidad y resistencia (Febré et al., 2015; Sánchez-Sánchez, Yagüe, Fernández, \& Petisco, 2014; SerraOlivares, González-Víllora, \& García-López, 2015). En balonmano, Balakrishnan, Rengasamy, y Aman (2011) emplearon el modelo tradicional de enseñanza frente al teaching games for understanding, utilizando situaciones de juego de 3vs.3 y encontrando mejoras estadísticamente significativas en la ejecución técnica y táctica. Resultado que concordó con los reportados por Morales y Arias-Estero (2015), los cuales observaron un aumento de la implicación y la percepción del esfuerzo en la situación de 4vs.4 frente al 7vs.7.

En baloncesto adulto también se estudió el efecto de la disminución del número de jugadores fundamentalmente para su aplicación a entrenamiento. McCornick et al. (2012) encontraron más contactos con el balón en la modalidad 3vs.3. Sampaio, Abrantes, y Leite (2009) compararon las modalidades 3vs.3 y 4vs.4, identificando diferencias a favor de la primera en cuanto al nivel de frecuencia cardíaca, esfuerzo percibido y en adquisición de potencia de salto, resultados que coincidieron con los obtenidos por Yanci, Iturricastillo, y Granados (2014) en relación a las demandas físicas de las situaciones reducidas en baloncesto. Clemente, GonzálezVíllora, Delextrat, Martins, y Vicedo (2017) comprobaron cómo las situaciones de 3vs.3 con diferentes objetivos favorecieron el aumento de la intensidad, volumen de juego y eficacia en los ataques frente a la forma tradicional de 5vs.5. Similares hallazgos obtuvieron Klusemann, Pyne, Foster, y Drinkwater (2012) respecto a la cantidad de sprints y en la frecuencia cardíaca en la modalidad 2vs.2 frente a 4vs.4. Delextrat y Martínez (2014) también encontraron mayores mejoras en la agilidad defensiva, técnica de tiro y fuerza de la parte superior del cuerpo empleando situaciones de entrenamiento de 2vs.2.

A pesar de los estudios encontrados en otros deportes en etapa de iniciación y en baloncesto adulto, en baloncesto de 9 a 11 años se ha investigado poco el efecto de la reducción del número de jugadores, porque los investigadores han prestado más atención a otros aspectos, fundamentalmente relacionados con los equipamientos (e.g., Arias-Estero et al., 2009, 2012, 2018). En dichas investigaciones no se comprobó el efecto sobre la participación de los jugadores o no se obtuvieron diferencias estadísticamente significativas que demostrasen una mayor posesión del balón. Por el contrario, Piñar (2005) y Piñar et al. (2009) encontraron que la modalidad 3vs.3 posibilitó más pases y más tiempo de posesión de balón a cada jugador en cada ataque. Tallir et al., (2012) observaron cómo dicha modalidad reducida produjo un aumento de las tomas de decisiones adecuadas, y la eficiencia de las ejecuciones técnicas. Por último, MartínezFernández et al. (2015) hallaron que la modalidad 3vs.3 posibilitó mayor número de posesiones de balón y más eficaces, así como un incremento en el número de situaciones de 1vs.1, en comparación con la modalidad 4vs.4.

A pesar que los cuatro estudios citados hasta ahora en esta introducción sugieren que la modalidad 3vs.3 posibilita mayores oportunidades de aprendizaje, ninguno de ellos fue realizado en contexto escolar, teniendo en cuenta las percepciones y preferencias de los protagonistas. Considerar las percepciones de los niños es fundamental para atender a sus demandas y posibilitar su motivación por la práctica deportiva (Arias-Estero et al., 2011b). El disfrute, competencia percibida y emociones son fundamentales para posibilitar la adherencia a la actividad deportiva (Brière, Vallerand, Blais, \& Pelletier, 1995; Fernández-Espínola \& Almagro, en prensa; Fraile-García, Tejero-González, Esteban-Cornejo, \& Veiga, 2019; Frederick \& Schuster-Schmidt, 2003; Pérez \& Guzmán, 2019). Estas se han asociado a conductas de práctica deportiva perdurables en el tiempo (Álvarez, Balaguer, Castillo, \& Duda, 2009; Fernández-Espínola \& Almagro, en prensa). Según la teoría del comportamiento planificado esto es posible porque la actitud influye sobre la intención de práctica deportiva futura como predictor más próximo para mantener el comportamiento (Ajzen, 1985).

El objetivo de esta investigación fue comprobar si la modalidad de juego 3vs.3, en comparación con la 5vs.5 en baloncesto extraescolar jugado por niños de entre 9 y 11 años, favoreció un aumento de: (a) número de posesiones de balón, (b) número de pases, (c) eficacia en las posesiones, (d) disfrute, (e) competencia percibida, (f) intención de práctica futura, (g) emociones positivas y (h) preferencia de los participantes. Atendiendo a los estudios de referencia en baloncesto y otros deportes, la primera hipótesis fue que los participantes obtendrían valores más elevados en la modalidad 3vs.3 en comparación con la 5vs.5 en: (a) número de posesiones de balón, (b) número de pases, y (c) eficacia en las posesiones. A partir de los resultados positivos esperados según la primera hipótesis, la segunda es que la modalidad 3vs.3 favorecería mayores puntuaciones en: (a) disfrute, (c) competencia percibida, (d) intención de práctica futura, y (e) emociones positivas. Además, la preferencia de los participantes sería mayor por la modalidad 3vs.3.

\section{Metodología}

\section{Participantes}

Participaron un total de 42 niños de entre 9 y 11 años ( $M$ $=9.89, D T=.832)$, que estaban inscritos en las actividad extraescolar de baloncesto en cuatro centros educativos diferentes situados en la Región de Murcia. Los participantes fueron seleccionados intencionadamente porque se comprometieron a cumplir los criterios de inclusión: (a) participar en todas las situaciones diseñadas, (b) completar los cuestionarios según el procedimiento explicado y (c) asistir a todas las sesiones. No obstante, debido a los criterios de inclusión exigidos, el estudio se realizó con 18 niños, que llevaban practicando baloncesto en horario extraescolar una media de 2.11 ( $D T=.75)$ años. Además, los niños practicaban a la semana una media de 2.17 horas $(D T=.38)$, durante una media de 2.17 días $(D T=.38)$. Ellos no se enfrentaban contra otros equipos hasta que comenzó el campeonato local, aproximadamente un mes después de finalizar el estudio. En todo caso, sus enfrentamientos fueron contra los equipos de otros centros educativos de la localidad. Ninguno de los participantes se lesionó durante el estudio. Los jugadores, entrenadores, padres y equipos directivos de los centros fueron informados sobre el estudio y aceptaron su ejecución. Para la participación de los jugadores se obtuvo el consentimiento informado de los padres. El estudio fue previamente aprobado por el Comité de Ética de la Universidad. 


\section{Diseño}

Se siguieron dos metodologías para llevar a cabo el trabajo. Por un lado, se utilizó la metodología observacional mediante un diseño de tipo ideográfico, seguimiento, multidimensional e intrasesional, con un muestreo temporal por puntos de tiempo (Anguera, 2003) para evaluar las dos modalidades de juego (5vs.5 y 3vs.3). El diseño fue ideográfico porque el grupo de participantes se estudió como unidad. Fue de tipo seguimiento puesto que se obtuvieron los datos a lo largo de siete mini-partidos de 8 minutos por cada modalidad de juego, durante siete sesiones, en el espacio de cuatro semanas consecutivas entre los meses de enero y febrero. Cada semana se desarrollaron dos sesiones (martes y jueves), a excepción de la última semana, en la cual solo se desarrolló una sesión. Los criterios de constancia intersesional fueron: (a) la hora de inicio y fin de las sesiones fue de 17:30 a 19:30, (b) las reglas fueron las oficiales de minibásquet, (c) la duración de cada partido fue de 8 minutos, (d) el espacio de juego fue de 14x15 m (mitad de pista), (e) la altura de la canasta fue de 2.60 m (una canasta en cada media pista), (f) el balón fue de talla 5, (g) el descanso entre los mini-partidos fue de 5 minutos, y (h) el árbitro fue un entrenador de minibásquet. Por último, el diseño fue multidimensional, porque el efecto de la modalidad de juego se comprobó sobre los siguientes criterios de diferente naturaleza: (a) número de posesiones de balón, (b) número de pases y (c) eficacia de las posesiones. Por otro lado, se empleó un diseño cuasi-experimental, a través del cual se comprobó el efecto de las dos modalidades de juego sobre: (d) disfrute, (e) competencia percibida, (f) intención de práctica futura y (g) estado emocional positivo neutro y negativo. Además, se analizó la preferencia de los participantes por la modalidad de juego así como los motivos que justificaran la elección.

\section{Procedimiento}

Los participantes asistieron a la instalación al menos 15 minutos antes del comienzo de las sesiones organizadas con motivo del estudio para prepararse. Esta era una instalación al aire libre. Realizaban un calentamiento a través del cual estiraban (7 minutos), movilizaban las articulaciones (7 minutos) y realizaban dos tareas de carrera y tiro (10 minutos). Posteriormente participaban en los enfrentamientos de las modalidades objeto de estudio aleatoriamente. Es decir, durante las siete sesiones, tres comenzaron jugando la modalidad 5vs.5 y cuatro con la modalidad 3vs.3. Los participantes fueron distribuidos aleatoriamente en los equipos, pero atendiendo al criterio de homogeneidad en cuanto al nivel de competencia motriz según sus entrenadores. Debido al número de participantes y para homogeneizar el tiempo de práctica dedicado a cada modalidad entre todos ellos, los equipos no fueron estables en cuanto a sus integrantes en ninguna de las modalidades. No obstante, atendiendo a su distribución, 14 participantes acumularon un tiempo de práctica de 64 minutos y 4 participantes de 56 minutos en la modalidad 5vs.5. Mientras que el tiempo en la modalidad 3vs.3 fue para todos de 56 minutos. Los participantes que tenían que esperar su turno para jugar realizaban tareas de bote y bote y tiro alternas, en una pista contigua. No obstante, en todas las sesiones se celebraron simultáneamente dos mini-parti- dos en pistas diferentes, cada uno en media pista. Los investigadores estuvieron presentes en todas las sesiones para controlar todas las cuestiones propias del estudio y resolver dudas tras las explicaciones de todos los procedimientos, incluidos los de toma de datos. Se les pidió a los participantes que afrontaran cada mini-partido como un partido de competición habitual. Por este motivo el tiempo de juego no se detuvo, como ocurre en el juego real, dado que no existieron pérdidas de tiempo deliberadas o prolongadas. No se realizaron cambios durante el tiempo de juego. La defensa individual fue obligatoria. Los participantes estaban familiarizados con el protocolo dado que la semana previa al comienzo del estudio se puso en práctica.

\section{Toma de datos}

Número de posesiones de balón, número de pases y eficacia de las posesiones. Los datos de los criterios número de posesiones de balón, número de pases y eficacia de las posesiones se obtuvieron a través de la filmación de los minipartidos. Esta se efectuó con dos cámaras (Everio Full GZHD7; JVC, Tokio, Japón) debido a la simultaneidad de los mismos. Cada una situada transversalmente a cada una de las pistas de juego para filmar a todos los participantes, el espacio y las canastas.

Una vez definidos los criterios (Figura 1), un observador fue entrenado durante 10 horas siguiendo la propuesta de Anguera (2003). Durante el adiestramiento se indicó al observador que la observación sería no participante, activa y directa y el registro sistemático (Anguera \& Blanco, 2003). Para incrementar la fiabilidad de la observación se establecieron las siguientes premisas: (a) cada ataque del equipo fue observado dos veces, (b) en la primera observación se observaban los criterios número de posesiones de balón y pases de cada participante, (c) en la segunda observación se observaba la eficacia de cada jugador, y (d) el observador no podía acumular más de 30 minutos continuos observando sin realizar un descanso de al menos 10 minutos. La observación fue de todos los participantes durante todos los minutos de juego. De cada participante se registró el número de veces que obtenía la posesión del balón, el número de pases que realizaba y la eficacia que implicaba la finalización de la posesión del balón durante todo el mini-partido. Al final del entrenamiento, se obtuvo la fiabilidad intraobservador tras haber observado un periodo de juego de 8 minutos y tras 10 días haber repetido la observación. El periodo de juego observado para obtener la fiabilidad fue diferente a los minipartidos del presente trabajo. La fiabilidad, obtenida a través del porcentaje de acuerdo, fue del 100\%.

El observador observó los siete mini-partidos de cada modalidad de juego dos veces, siguiendo las premisas descritas anteriormente. De modo que primero observó los 14 mini-partidos y tras 10 días de no observación los volvió a

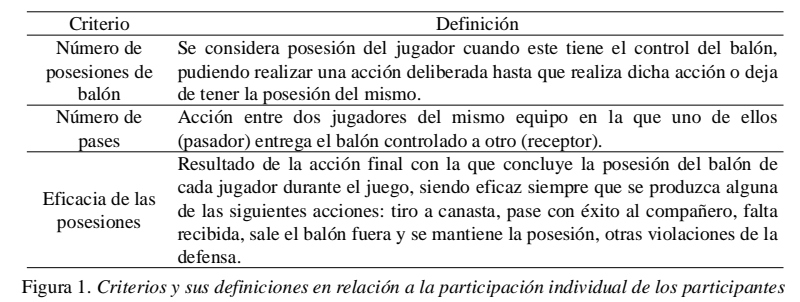


observar en el mismo orden. La fiabilidad intraobservador en este caso fue del $100 \%$ en los criterios número de posesiones de balón y número de pases, pero del $90 \%$ en el criterio eficacia de las posesiones.

Disfrute y competencia percibida. El disfrute y la competencia percibida se obtuvieron a través de la escala validada por Arias-Estero, Alonso, y Yuste (2013). Esta escala cuenta con siete ítems referidos al disfrute practicando el deporte (á de Cronbach $=.89$ ) y al sentimiento o consideración con respecto a uno mismo de su competencia practicando baloncesto (á de Cronbach $=.89$ ). La escala de respuesta fue tipo Likert de 1 a 5 , siendo 1 «totalmente en desacuerdo» y 5 «totalmente de acuerdo». Los participantes completaron este instrumento al final de cada una de los 14 mini-partidos. Para ello emplearon un tiempo inferior a 5 minutos.

Intención de práctica futura. La intención de práctica futura se obtuvo utilizando la escala validada por Arias-Estero, Castejón, y Yuste (2013). Esta escala cuenta con cinco ítems referidos a su propósito de seguir practicando baloncesto en el futuro (á de Cronbach $=.90$ ). La escala de respuesta fue tipo Likert de 1 a 5 , siendo 1 «totalmente en desacuerdo» y 5 «totalmente de acuerdo». Los participantes completaron este instrumento al final de cada una de los 14 mini-partidos y tras contestar a la escala previa. Para ello emplearon un tiempo inferior a 5 minutos.

Estado emocional. Los datos referentes al estado emocional suscitado se recogieron a través de la Games and Emotions Scale (Lavega, March, \& Filella, 2013). El instrumento está compuesto por un total de 13 emociones, cuatro de ellas positivas (alegría, afecto, humor y felicidad, á de Cronbach $=.88$ ), seis negativas (tristeza, miedo, ansiedad, ira, rechazo y vergüenza, á de Cronbach $=.85$ ), y tres neutras o ambiguas (sorpresa, esperanza y compasión, á de Cronbach $=.84$ ). Cada una de ellas está acompañada de una escala tipo Likert de 0 a 10, siendo 0 nada intenso y 10 muy intenso. Los participantes completaron este instrumento al final de cada una de los 14 mini-partidos y tras contestar las escalas previas. Para ello emplearon un tiempo inferior a 7 minutos.

Preferencia por la modalidad de juego. La preferencia por la modalidad de juego se recogió por escrito empleando dos preguntas ad hoc. La primera fue cerrada con dos opciones de respuesta: «¿Con qué modalidad de juego prefieres jugar a baloncesto (5vs.5 o 3vs.3)?» La segunda fue abierta: «Indica el motivo más importante por el que prefieres jugar a baloncesto de esa manera». Los participantes contestaron ambas preguntas al final de cada una de las siete sesiones y tras contestar a las escalas previas. Para ello emplearon un tiempo inferior a 3 minutos.

\section{Análisis de datos}

El análisis de los datos se realizó mediante el programa estadístico IBM SPSS Statistics v.20.0 para Windows. Los datos de los participantes fueron analizados como medias y desviaciones típicas de número de posesiones de balón, número de pases, eficacia de las posesiones, disfrute, competencia percibida, intención de práctica futura, emociones positivas, negativas y neutras. En primer lugar se comprobó que los datos siguieron una distribución normal con la prueba de Kolmogorov-Smirnov y se obtuvo que los datos fueron paramétricos. Se utilizó la prueba t de Student para cono- cer las diferencias entre ambas modalidades en cuanto a los criterios anteriormente indicados. La preferencia de los participantes así como sus motivos se extrajeron directamente de las dos preguntas escritas y se obtuvo su recuento y porcentaje relativo a la modalidad de juego y comparando entre modalidades de juego. Los motivos fueron agrupados bajo las categorías que emergieron directamente de las respuestas de los participantes. Por último, se utilizó la prueba t de Student para comprobar los posibles efectos de la aleatorización de la modalidad de juego, así como para hacer lo propio entre los participantes que habían acumulado más minutos de práctica y sus compañeros con menor tiempo en la modalidad 5vs.5. El nivel estadístico de significación se estableció con $p<.05$ en todas las pruebas. Se calculó el tamaño del efecto $(d)$ para todos los criterios.

\section{Resultados}

Los resultados mostraron diferencias estadísticamente significativas entre las dos modalidades de juego con respecto al número de posesiones de balón, número de pases, eficacia de las posesiones, estado emocional positivo y neutro. Esto es, la modalidad 3vs.3 posibilitó valores superiores en dichos criterios. Además, los resultados fueron ratificados por los altos valores de tamaño de efecto al encontrarse entre .717 y 1.431 (Tabla 1). No obstante, en los criterios que no se obtuvieron diferencias estadísticamente significativas, el tamaño del efecto también fue entre alto y muy alto positivamente a favor de la modalidad 3vs.3.

Los participantes expresaron una mayor preferencia por la modalidad 3vs.3 que por la modalidad 5vs.5 (85.71\% vs. 14.29\%, Tabla 2). Los motivos fundamentales que justificaron dicha elección estuvieron asociados a la participación. Estos fueron, porque mi equipo ha tenido más el balón (100\% vs. $0 \%$ ), porque la he pasado más ( $100 \%$ vs. $0 \%)$, y porque he tocado más el balón (94.74\% vs. 5.26\%). Por el contrario, en la modalidad 5vs.5 predominaron respuestas asociadas al resultado (porque he ganado: $33.33 \%$ ), disfrute (porque me lo pasé bien: $22.22 \%$ y competencia percibida (porque he jugado bien: 22.22\%), aunque el porcentaje de respuestas también fue mayor en la modalidad 3vs.3 en todas ellas, excepto en la relativa al resultado (Tabla 2).

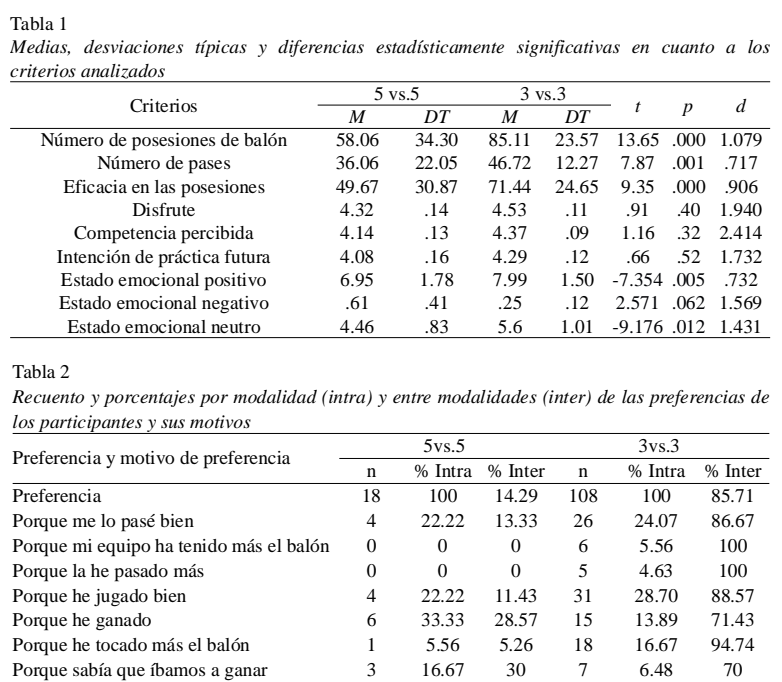


No se encontraron diferencias estadísticamente significativas al comprobar los posibles efectos de la aleatorización de la modalidad de juego, ni en cuanto al tiempo de práctica acumulado en la modalidad 5vs.5 ( $p>.05)$.

\section{Discusión}

El objetivo de esta investigación fue comprobar si la modalidad de juego 3vs.3, en comparación con la 5vs.5 en baloncesto extraescolar jugado por niños de entre 9 y 11 años, favoreció un aumento de: (a) número de posesiones de balón, (b) número de pases, (c) eficacia en las posesiones, (d) disfrute, (e) competencia percibida, (f) intención de práctica futura, (g) emociones positivas y (h) preferencia de los participantes. Los resultados ratificaron la primera hipótesis y parcialmente la segunda, puesto que en la modalidad 3vs.3 se encontraron valores más elevados en: (a) número de posesiones de balón, (b) número de pases, (c) eficacia de las posesiones, (d) emociones positivas y (e) emociones neutras. Al mismo tiempo, la preferencia de los participantes por dicha modalidad fue ratificada por sus percepciones subjetivas. Sin embargo, no existieron diferencias estadísticamente significativas entre modalidades con respecto a: (a) disfrute, (c) competencia percibida, (d) intención de práctica futura, y (e) emociones negativas. En general, estos resultados apuntaron a que la modalidad reducida fue más adecuada que la estandarizada porque posibilitó una mayor participación en contacto con el balón y más eficaz, como sugirieron las propuestas teóricas (e.g., American Sport Education Program, 2006; Giménez \& Sáenz-López, 2004; Mitjana, 2000) y las evidencias hasta ahora conocidas (Martínez-Fernández et al., 2015; Piñar, 2005; Piñar et al., 2009; Tallir et al., 2012). Pero además, la modalidad 3vs.3 posibilitó un aumento de las emociones positivas y neutras, sin observarse ningún efecto negativo sobre criterios asociados a la motivación hacia el deporte. Por lo que dicha modalidad fue preferida por los participantes y en consecuencia favorecería una práctica deportiva más prolongada en el tiempo (Palomäki et al., 2018).

Al igual que ocurrió en el estudio de Martínez-Fernández et al. (2015), los participantes del presente trabajo también obtuvieron en más ocasiones la posesión del balón en la modalidad 3vs.3. Este resultado también coincidió con el hallado por Piñar et al. (2009), los cuales observaron un mayor tiempo de posesión del balón de cada jugador en la modalidad 3vs.3. En la misma línea, la implicación también fue mayor en estudios previos realizados en otros deportes de iniciación (Da Silva et al., 2011; Morales \& Arias-Estero, 2015) y en baloncesto adulto (McCornick et al., 2012; Sampaio et al., 2009; Yanci et al., 2014). De ahí, que dos de los tres motivos más valorados por los participantes para preferir dicha modalidad en el presente trabajo fueron porque su equipo había obtenido el balón en un mayor número de ocasiones y porque individualmente también consiguieron la posesión del móvil más veces (Tabla 2). Por el contrario, los resultados fueron opuestos a los reportados por Arias-Estero et al. (2011a), los cuales no encontraron diferencias al implementar un balón de menor masa, tras lo que sugirió la importancia de la disminución del número de jugadores para adecuar el juego a las posibilidades de los jugadores. La práctica en contacto con el balón resulta muy significativa para el jugador porque le permite practicar acciones de juego en su posesión a la vez que le motiva por poseer el elemento de juego que centra la atención del resto de participantes (Piñar, Cárdenas, Conde, Alarcón, \& Torre, 2007). De modo que ambos hechos son muy positivos para que el jugador aprenda a jugar un deporte (Schmidt \& Lee, 2005).

Junto con el incremento en el número de posesiones de balón, también aumentó el número de pases en la modalidad 3vs.3, como en los estudios de Piñar (2005) y Arias-Estero et al. (2012) tras reducir el número de jugadores participantes y jugar con un balón de menor masa, respectivamente. Igualmente, el número de pases también aumentó en fútbol de iniciación y en baloncesto adulto al analizar el efecto de la disminución del número de jugadores (Da Silva et al., 2011; Katis \& Kellis, 2009; Klusemann et al., 2012). Además, pasar más el balón fue otro de los tres motivos fundamentales por el que los participantes del presente estudio prefirieron en mayor medida la modalidad 3vs.3 (Tabla 2). El pase es una acción básica en los deportes de equipo porque posibilita interaccionar a través del balón con el resto de integrantes del equipo a la vez que su uso adecuado durante el juego es fuente de incertidumbre para el rival (Cárdenas \& Pintor, 2001; Giménez \& Sáenz-López, 2004; Klusemann et al., 2012). Que los participantes del presente estudio realizasen más pases en la modalidad 3vs.3 evidencia un estilo de juego más activo e intenso, como se ha demostrado en estudios previos (Clemente et al., 2017; McCornick et al., 2012; Sampaio et al., 2009; Yanci et al., 2014). Dicho estilo favorece el tiro a canasta y es recomendado en baloncesto de 9 a 11 años (American Sport Education Program, 2006; Arias-Estero et al., 2012; Piñar, 2005).

En el presente trabajo también aumentó la eficacia de las posesiones de los participantes en la modalidad 3vs.3, al igual que ocurrió en los trabajos encontrados en baloncesto de 9 a 11 años (Martínez-Fernández et al., 2015; Tallir et al., 2012), baloncesto adulto (Clemente et al., 2017; Delextrat y Martínez, 2014) y otros deportes de iniciación deportiva (Balakrishnan et al., 2011; Febré et al., 2015; Sánchez-Sánchez et al., 2014; Serra-Olivares et al., 2015). Esto pudo ocurrir porque en la modalidad reducida disminuyó la dificultad del juego en cuanto a toma de decisión, dado que en el mismo espacio participaron seis jugadores en lugar de diez. Al disponer de más espacio libre entre atacantes y defensores y entre el poseedor del balón y su defensor directo las acciones resultan más fáciles y posibilitan mejores decisiones que cuando el espacio disponible es más reducido (Araújo, Davids, Bennett, Button, \& Chapman, 2004; Cantos \& Moreno, en prensa; Menuchi, Moro, Ambrósio, Pariente, \& Araújo, 2018). Es por ello que Martínez-Fernández et al. (2015) también encontraron un aumento de las situaciones de 1vs.1 en la modalidad más reducida. La práctica con éxito es un indicador del aprendizaje de las acciones motrices, de modo que cuando los niños obtienen éxito en su práctica ellos mejoran y consolidan sus aprendizajes (Ashy, Lee, \& Landin, 1988; Buck, Harrison, \& Bryce, 1990). Es por ello que posibilitar que se obtenga éxito con frecuencia durante el juego, sin que este deje de suponer un reto, es una estrategia pedagógica poderosa para generar afiliación al deporte a la vez que favorecer el aprendizaje (Deci \& Ryan, 1985).

En relación a la motivación, como sucedió en el estudio 
de Morales y Arias-Estero (2015) en balonmano extraescolar, los resultados con respecto a disfrute, competencia percibida e intención de práctica futura no mostraron diferencias estadísticamente significativas al comparar ambas modalidades de juego. No obstante, aunque los valores hallados fueron mayores en la modalidad 3vs.3, en general fueron ligeramente inferiores a los encontrados en jugadores de baloncesto federado con edades entre 9 y 17 años (Almagro \& Conde, 2012; Arias-Estero et al., 2013; Franco et al., 2012). Sin embargo, los participantes indicaron que preferían la modalidad reducida en mayor medida que la reglamentaria porque «se lo pasaron bien» y porque «habían jugado bien» (Tabla 2). Esto pudo deberse a que los participantes de los estudios mencionados competían a nivel federado mientras que los del presente estudio practicaban baloncesto como actividad extraescolar. Esta diferencia puede influir en los resultados como se ha reportado en trabajos previos (Moreno, Hellín, González-Cutre, \& Martínez-Galindo, 2011). Por otro lado, pudo ser que las diferencias entre modalidades en el presente trabajo no fueran mayores porque los niños participaron de manera voluntaria. Según Deci y Ryan (1985) este es un factor que influye en que las valoraciones de los participantes se mantuvieran elevadas.

Los resultados del presente trabajo coincidieron con los encontrados por Serna (2014), dado que la situación modificada, en este caso la modalidad 3vs.3, no generó más emociones negativas. Además, en el presente estudio, la modalidad 3vs.3 posibilitó que aumentaran las emociones positivas y neutras. Ambos resultados pudieron estar ligados a cuestiones relativas a la cooperación y coordinación entre compañeros, así como al resultado de la misma. En otras palabras, la modalidad $5 \mathrm{vs.} 5$ genera mayores fuentes de conflicto entre los jugadores que la 3vs.3 (Sáez de Ocáriz \& Lavega, 2013, 2014), pues demanda una mayor exigencia coordinativa con los compañeros del equipo (Bourbousson, Poizat, Saury, \& Seve, 2010). Por otro lado, en la modalidad 3vs.3 se produjo una mayor interacción motriz entre jugadores, como así demostraron los resultados relativos a número de posesiones de balón y pases (Tabla 1) y las percepciones de los participantes (Tabla 2), lo que genera emociones positivas más intensas, que además fueron reforzadas por el incremento de la eficacia en dicha modalidad (Lavega et al., 2013).

\section{Conclusión}

En conclusión, la modalidad 3vs.3 en el ámbito extraescolar posibilitó una mayor participación en contacto con el balón y con mayor éxito que la modalidad 5vs.5, favoreciendo la aparición de emociones positivas y neutras. Lo que fue reforzado al no observarse diferencias en los criterios disfrute, competencia percibida, intención de práctica futura y emociones negativas. Por lo que para a favorecer la implicación en el juego, una práctica de calidad, las emociones positivas, y a la vez satisfacer las preferencias de los niños, los entrenadores, profesorado y responsables de las competiciones de baloncesto entre 9 y 11 años deberían optar por dicha modalidad de juego. No obstante, los resultados del presente trabajo deben ser considerados con precaución debido al carácter descriptivo del diseño y a las características de los participantes. De modo que en futuras investigaciones se tendrían que confirmar los resultados obtenidos en la presente mediante diseños más complejos. Además, es preciso estudiar el efecto de la modalidad de juego sobre otras variables de carácter fisiológico, así como conocer la percepción de los participantes y entrenadores o profesorado al respecto.

\section{Referencias}

Ajzen, I. (1985). From intention to actions: A theory of planned behavior. En J. Kuhl y J. Beckmann (Eds.), Action-control: From cognition to behaviour (pp. 11-39). Heidelberg, Alemania: Springer.

Almagro, B., \& Conde, C. (2012). Factores motivacionales como predictores de la intención de ser físicamente activos en jóvenes jugadores de baloncesto. Cuadernos de Psicología del Deporte, 12, 1-4.

Álvarez, M. S., Balaguer, I., Castillo, I., \& Duda, J. (2009). Coach autonomy support and quality of sport engagement in young soccer players. The Spanish Journal of Psychology, 12, 138-148.

American Sport Education Program. (1996). Coaching youth basketball ( $2^{\text {nd }}$ ed.). Champaign, IL: Human Kinetics.

Anguera, M. T. (2003). La observación. En C. Moreno Rosset (Ed.), Evaluación psicológica. Concepto, proceso y aplicación en las áreas del desarrollo y de la inteligencia (pp. 271-308). Madrid: Sanz y Torres.

Anguera, M. T., \& Blanco, A. (2003). Registro y codificación en el comportamiento deportivo. En A. Hernández Mendo (Coord.), Psicología del Deporte (Vol. 2). Metodología (p. 6-34). Buenos Aires, Argentina: Tulio Guterman (www. efdeportes.com).

Araújo, D., Davids, K., Bennett, S. J., Button, C., \& Chapman, G. (2004). Emergence of sport skills under constraints. In M. Williams, \& N. Hodges (Eds.), Skill acquisition in sport: Research, theory and practice (pp. 409-433). New York, NY: Routledge.

Arias-Estero, J. L., Argudo, F., \& Alonso, J.I. (2009). Effect of the 3- point line change on the game dynamics in girls' minibasketball. ResearchQuarterlyforExercise and Sport, 80, 502-509.

Arias-Estero, J. L., Argudo, F. M., \& Alonso, J. I. (2011a). Number of players who gain ball possession as a function of basketball mass among 9-11-year-old male players. Perceptual and Motor Skills, 113(2), 557-562.

Arias-Estero, J. L., Argudo, F. M., \& Alonso, J. I. (2011b). Review of rule modification in sport. Journal of Sports Science and Medicine, 10, 1-8.

Arias-Estero, J. L., Argudo, F. M., \& Alonso, J. I. (2012). Effect of ball mass on dribble, pass, and pass reception in 9-11-year-old boys' basketball. Research Quarterly for Exercise and Sport, 83, 407-412.

Arias-Estero, J. L., Argudo, F. M., \& Alonso, J. I. (2018). Oneon-one situation decision-making according to equipment in youth basketball. International Journal of Sports Science \& Coaching, 13, 72-77.

Arias-Estero, J. L., Alonso, J. I., \& Yuste, J. L. (2013). Propiedades psicométricas y resultados de la aplicación de la escala de disfrute y competencia percibida en balonces- 
to de iniciación. UniversitasPsychologica, 12, 945-957.

Arias-Estero, J. L., Castejón, F. J., \& Yuste, J. L. (2013). Propiedades psicométricas de la escala de intencionalidad de ser físicamente activo en Educación Primaria. Revista de Educación, 362, 485-505.

Ashy, M. H., Lee, A. M. \& Landin, D. K. (1988). Relationship of practice using correct technique to achievement in a motor skill. Journal of Teaching in Physical Education, 7,115-120.

Balakrishnan, M., Rengasamy, S., \&Aman, M. S. (2011). Effect of teaching games for understanding approach on student's cognitive learning outcome. World Academy of Science, Engineering and Technology, 77, 961-963.

Bourbousson, J., Poizat, G., Saury, J., \& Seve, C. (2010). Team coordination in basketball: Description of the cognitive connections among teammates. Journal of Applied Sport Psychology, 22, 150-166.

Brière, N., Vallerand, R., Blais, M., \& Pelletier, L. (1995). Development and validation of a measure of intrinsic, extrinsic and amotivation in sports: The sport motivation scale (SMS). International Journal of Sport Psychology, 26(4), 465-489.

Buck, M., Harrison, J. M., \& Bryce, G. R. (1990). An analysis of learning trials and their relationship to achievement in volleyball. Journal of Teaching in Physical Education, 10,134-152.

Cantos, J., \& Moreno, F. J. (en prensa). Pedagogía no lineal como método de enseñanza de los comportamientos tácticos en los deportes de equipo, aplicación al rugby. $R e-$ tos.

Cárdenas, D., \& Pintor, D. (2001). La iniciación al baloncesto en el medio escolar. En F. Ruiz, A. García y A.J. Casimiro. (Eds.), La iniciación deportiva basada en los deportes colectivos: nuevas tendencias metodológicas (pp.105144). Madrid, España: Gymnos.

Carrillo, A., \& Rodríguez, J. (2004). El básquet a su medida: Escuela de básquet de 6 a 8 años. Barcelona, España: Inde.

Carron, A. V., Widmeyer, W. N., \& Brawley, L. R. (1989). Perception of ideal group size in sport teams. Perceptual and Motor Skills, 69, 1368-1370.

Clemente, F. M., González-Víllora, S., Delextrat, A., Martins, F. M., \& Vicedo, J. C. (2017). Effects of the sports level, format of the game and task conditionon heart rate responses, technical and tactical performance of youth basketball players. Journal of Human Kinetics, 58, 141155.

Da Silva, C. D., Impellizzeri, F. M., Natali, A. J., de Lima, J. R., Bara-Filho, M. G, Silami-Garçia, E., \& Marins, J. C. (2011). Exercise intensity and technical demands of small-sided games in young Brazilian soccer players: Effect of number of players, maturation, and reliability. The Journal of Strength \& Conditioning Research, 25, 2746-2751.

Deci, E. L., \& Ryan, R. M. (1985). The general causality orientations scale: Self-determination in personality. Journal of Research in Personality, 19, 109-134.

Delextrat, A. \& Martínez,A. (2014). Small-sided game training improves aerobic capacity and technical skills in basketball players. International Journal of Sport Medicine, 35, 385-391.
Febré, R., Chirosa, L. J., Casamichana, D., Chirosa, I., MarínTamallo, I., \& Pablos, C. (2015). Influencia de la densidad de jugadores sobre la frecuencia cardíaca y respuestas técnicas en jóvenes jugadores de fútbol. RICYDE. Revista Internacional de Ciencias del Deporte, 40, 116128.

Fernández-Espínola, C., \& Almagro, B. J. (en prensa). Relación entre motivación e inteligencia emocional en Educación Física: Una revisión sistemática. Retos.

Fraile-García, J., Tejero-González, C. M., Esteban-Cornejo, I., \& Veiga, O. L. (2019). Asociación entre disfrute, autoeficacia motriz, actividad física y rendimiento académico en educación física. Retos, 36, 58-63.

Franco, E., Pérez-Tejero, J., \& Arrizabalaga, A. (2012). Motivación e intención de ser físicamente activo en jugadores de baloncesto en formación. Diferencias en función de la competición. Cuadernos de Psicología del Deporte, 12(Supl. 1), 23-26.

Frederick, C. M., \& Schuster-Schmidt, H. (2003). Competition and intrinsic motivation in physical activity: Acomparison of two groups. Journal of Sport Behavior, 26, 240-254.

Giménez, F. J., \& Sáenz-López, P. (2004). Aspectos teóricos y prácticos de la iniciación al baloncesto. Sevilla, España: Wanceulen.

Katis, A., \& Kellis, E. (2009). Effects of small-sided games on physical conditioning and performance in young soccer players. Journal of Sports Science \& Medicine, 8, 374.

Klusemann M., Pyne D., Foster C., \& Drinkwater (2012). Optimising technical skills and physical loading in smallsided basketball games. Journal of Sports Sciences, 30, 1463-1471.

Lavega, P., March, J., \& Filella, G. (2013). Juegos deportivos y emociones. Propiedades psicométricas de la escala GES para ser aplicada en la Educación Física y el Deporte. Revista de Investigación Educativa, 31, 151-165.

Martínez-Fernández, S., García, J., \& Ibáñez, S. J. (2015). Incidence of type of game mode in player participation in minibasket. Revista de Psicología del Deporte, 24, 6568.

Mayol, M. T., Leon, M. T., \& Cardenas, D. (2016). The threepoint line and its influence on the gameplay in minibasketball, the degree of individual satisfaction and perceived competence of children in the Region of Murcia. Sport tk-revista Euroamericana de Ciencias del Deporte, 5, 89-101.

McCornick, B. T., Hannon, J. C., Newton, M., Shultz, B., Miller, N., \& Young, W. (2012). Comparison of Physical Activity in small-sided basketball games versus full-sided games. International Journal of Sports Science \& Coaching, 7, 689-697.

Menuchi, M. R. T. P., Moro, A. R. P., Ambrósio, P. E., Pariente, C. A. B., \& Araújo, D. (2018). Effects of spatiotemporal constraints and age on the interactions of soccer players when competing for ball possession. Journal of Sports Science and Medicine, 17, 379-391.

Mitjana (2000). Nuevas reglas. Clinic: Revista Técnica de Baloncesto, 50, 42-43.

Morales, M. T., \& Arias-Estero, J. L. (2015). Diferencias entre el juego 7 vs. 7 y el 4 vs. 4 en el balonmano escolar en relación al rendimiento, percepción del esfuerzo y la 
intencionalidad de práctica. Retos, 27, 34-39.

Moreno, J. A., Hellín, P., González-Cutre, D., \& MartínezGalindo, C. (2011). Influence of perceived sport competence and body attractiveness on physical activity and other healthy lifestyle habits in adolescents. Spanish Journal of Psychology, 14, 282-292.

Palomäki, S., Hirvensalo, M., Smith, K., Raitakari, O., Männistö, S., Hutri Kähönen, N., \& Tammelin, T. (2018). Does organized sport participation during youth predict healthy habits in adulthood? A 28 year longitudinal study. Scandinavian Journal of Medicine \& Science in Sports, 28, 1908-1915.

Pérez, D., \& Guzmán, J. F. (2019). Predictores cognitivos de la intención de práctica y la percepción de las relaciones en el deporte: Análisis de la pasión como mediadora. Retos, 36, 193-202.

Piñar, M. I. (2005). Incidencia del cambio de un conjunto de reglas de juego sobre algunas de las variables que determinan el proceso de formación de los jugadores de minibasket (9-11 años). Granada: Universidad de Granada.

Piñar, M. I., Cárdenas, D., Alarcón, F., Escobar, R., \& Torre, E. (2009). Participation of mini-basketball players during small-sided competitions. Revista de Psicología del Deporte, 18(3), 445-449.

Piñar, M. I., Cárdenas, D., Conde, J., Alarcón, F., \& Torre, E. (2007). Satisfaction in minibasketball players. Iberian Congress on Basketball Research, 4, 122-125.

Sáez de Ocáriz, U., \& Lavega, P. (2013). Transformar conflictos en educación física en primaria a través del juego. Aplicación del índice de conflictividad. Cultura y Educación, 25, 549-560.

Sáez de Ocáriz, U., \& Lavega, P. (2014). Hacia una transfor- mación de los conflictos motores en Educación Física. Cultura_Ciencia_Deporte, 9, 43-55.

Sampaio, J., Abrantes, C., \& Leite, N. (2009). Power, heart rate and perceived exertion responses to $3 \times 3$ and $4 \times 4$ basketball small-sided games. Revista de Psicología del Deporte, 18(3), 463-467.

Sánchez-Sánchez, J., Yagüe, J. M., Fernández, R. C., \& Petisco, C. (2014). Efectos de un entrenamiento con juegos reducidos sobre la técnica y la condición física de jóvenes futbolistas. RICYDE. Revista Internacional de Ciencias del Deporte, 37, 221-234.

Schmidt, R. A., \& Lee, T. D. (2005). Motor control and learning: A behavioral emphasis (4th ed.). Champaign, IL: Human Kinetics.

Serna, J. (2014). Inteligencia motriz e inteligencia emocional en el baloncesto. Lleida, España: Universidad de Lleida.

Serra-Olivares, J., Gonzalez-Villora, S., \& Garcia-Lopez, L. M. (2015). Effects of modification of task constrains in 3versus-3 small-sided soccer games. South African Journal for Research in Sport, Physical Education and Recreation, 37, 119-129.

Tallir, I. B., Phillipaerts, R., Valcke, M., Musch, E., \& Leonor, M. (2012). Learning opportunities in 3 on 3 versus 5 on 5 basketball game play: An application of non linear pedagogy. International Journal of Sport Psychology, 43, 420-437.

Tous, J. (1999). Reglamento de baloncesto comentado. Barcelona, España: Paidotribo.

Yanci, J., Iturricastillo A., \& Granados, C. (2014). Heart rate and body temperature response of wheelchair basketball players in small-sided games. International Journal of Performance Analysis in Sport, 14, 535-544.

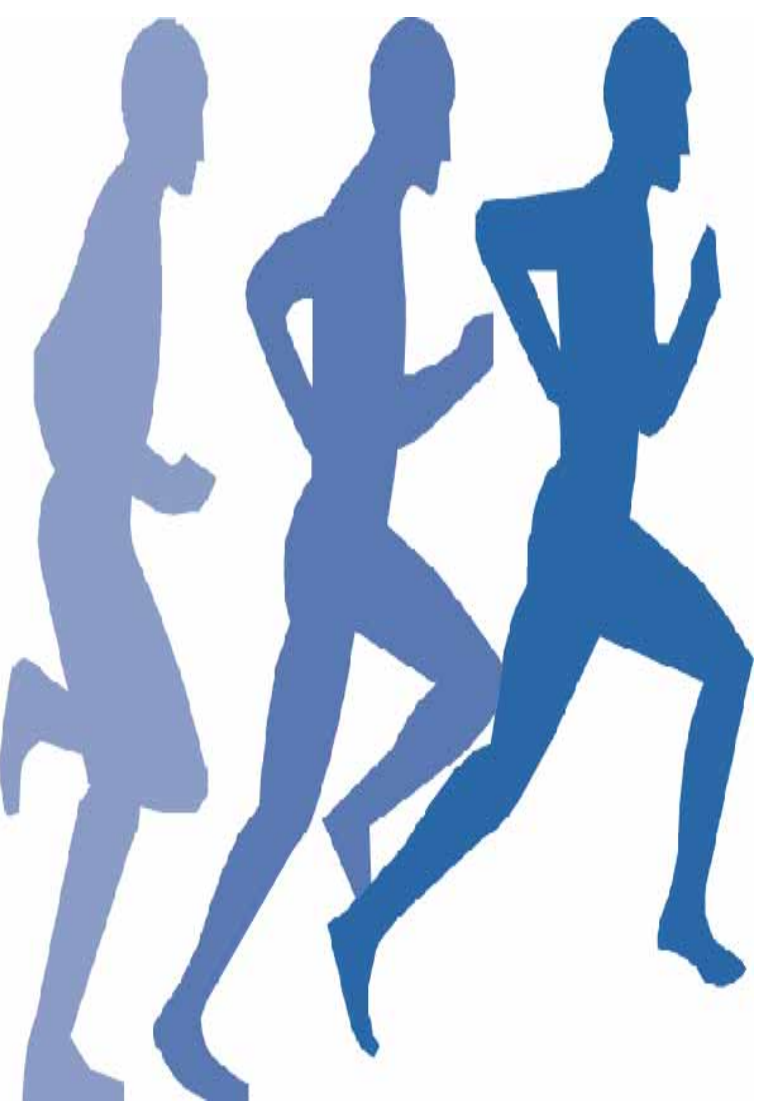

